\title{
Evolución natural del prepucio
}

\author{
Dr. Humberto Soriano B.; Dr. Miguel O’Ryan G.; Dr. Jorge Torres P.; Dr. Enrique Fanta N.; \\ Dra. A ma Muñoz M.; Dr. Humberto Sorjano P.l
}

\section{Normal foreskin development}

\begin{abstract}
A total of 1.968 children from 0 to 21 years old, were examined to determine the characteristics of the foreskin. Four main morphological types were found in the prepuce: "normal" (not tight nor adhered), phymotic (stenotic, not retractile), tight (retractile but stenotic) adhered (balano prepucial adherence). Less than $20 \%$ of children 0 to 23 months had the "normal" type, predominating the adherent and phymotic forms. During chibhood and adolescente there is a natural evolution towards the "normal" type, which was present in $50 \%$ of seven year old and in $95 \%$ of 18 year old boy. The authors state that these morphologies are part of the normal foreskin development and recommena not to interfer with it unless there is a concomitant pathology.

(Key words: Prepuce, foreskin, normal development).
\end{abstract}

No existen estudios nacionales referentes al desarrollo prepucial ${ }^{1}$. Los estudios extranjeros no cubren un rango etario amplio o bien, analizan poblaciones reducidas. Esta falta de conocimiento plantea el dilema de la conducta a adoptar con el prepucio del niño cuando su morfologia no corresponde a la forma tipo adulto habitual. Con este trabajo, se pretende establecer las características del prepucio en las djstintas edades, para así determinar la evolución natural del desarrollo clínico morfológico prepucial.

\section{MATERIAL Y METODOS}

Se examinaron niños varones de 0 a 21 años, provenientes del Centro de diagnóstico de la Universidad Católica (CEDLUC), salas cunas de la Junta macional de jardines infantiles (JUNJI), escuelas y liceos estatales, reclutas de las fuerzas armadas (FF.AA.) y consultas de médicos participantes.

El examen realizado por médicos e internos, previamente instruidos, consistió en una retracción prepucial suave, firme no traumática, para observar espacio balano prepucial $y$ glande. En uла hoja ad hoc se tabularon: datos personales, antecedentes de patología balano prepucial, circuncisión y se esquematizó el hallazgo del examen.

\section{RESULTADO}

Se estudiaron 1.968 sujetos: 383 lactantes (0 a 23 meses), 536 preescolares ( 2 a 5 años), 454

1. Departamento de pediatría, Escuela de Medicina, Pontificia Universidad Católica de Chile. escolares ( 6 a 12 años) y 595 jóvenes y adolescentes (13 a 21 años). La observación permitió clasificar la morfología prepucial en 4 grupos (figura 1):

Fimosis: cubierta no retráctil, puede o no visualizarse el meato. Prepucio estrecho: retráctil, forma un cuello tras el surco balano prepucial que puede ser leve, moderado o severo, dependiendo de la magnitud de la estrechez y de la dificultad para đevolver el prepucio a su posición original. Pre. pucio adherido: prepucio adherido al glande en su tercio proximal solamente (leve), tercio medio (moderada), hasta el tercio distal (severo). Prepucio normal: tipo adulto en donde el prepucio es fácilmente retràctil, sis adherencia balano prepucial. Las morfologías descritas se podrían presentar en forma combinada ( $\mathrm{Ej}$.: estrecho y adherido).

De acuerdo con el criterio anterior, se encontró fimosis en $35 \%$ de los lactantes (primordialmente en los menores de un año, donde $50 \%$ de los prepucios son fimóticos), disminuyendo a $8 \%$ en el preescolar y luego gradualmente hasta $2 \%$ a los 10 años y $1 \%$ desde los 18 años en adelante (fig. 2). La estrechez prepucial se presentó en cerca de $25 \%$ de los menores de 2 años, disminuyendo en forma progresiva hasta $5 \%$ en la adolescencia. En estos últimos fue, en su gran mayoría, de carácter leve, no refiriendo los jóvenes molestia a causa de ella. Las adherencias, están presentes en cerca de la mitad de los menores de dos años, predominando la forma severa. Estas se mantienen durante el periodo preescolar aungue tienden a ir modificándose hacia la forma leve. Posteriormente se observó 


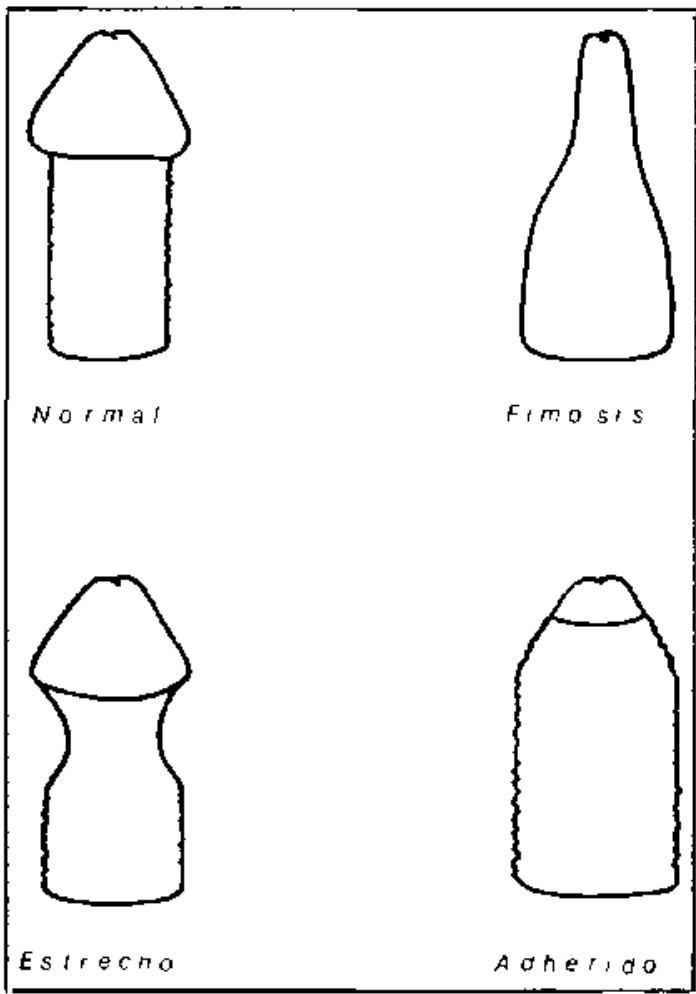

Figura 1: Clasiticación de la morlologia prepucial.

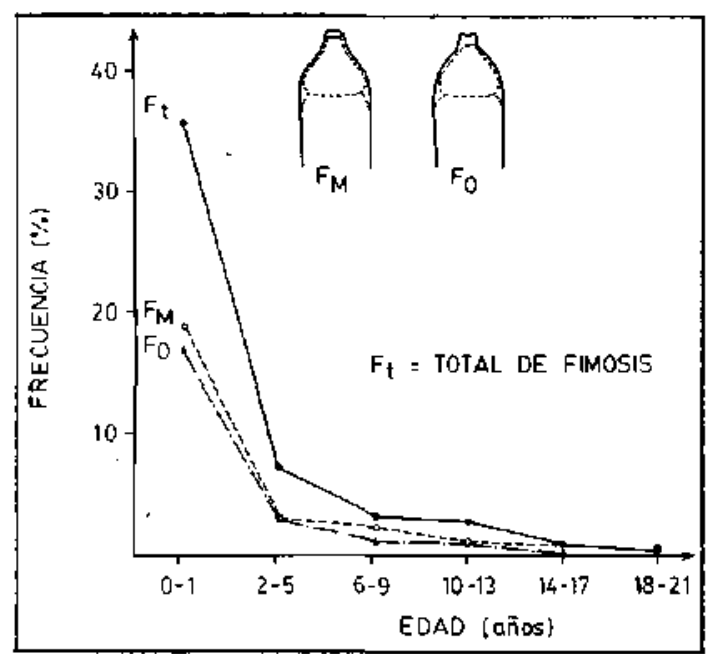

Figura 2: Evolución de la fïmosis.

progresiva disminución de su frecuencia, llegando a $20 \%$ a los 10 años, para desaparecer a los 21 años $(0 \%)$. Las formas severas desaparecen después de los 14 años (fig. 3 ).

La fig. 4 resume la evolución de las distintas morfologías prepuciales. La forma considerada "normal" muestra una curva sigmoídea, presen. tándose en $18 \%$ de los menores de 23 meses, $50 \%$ de los niños a los 7 años, y en $95 \%$ de ellos a los 18 años.

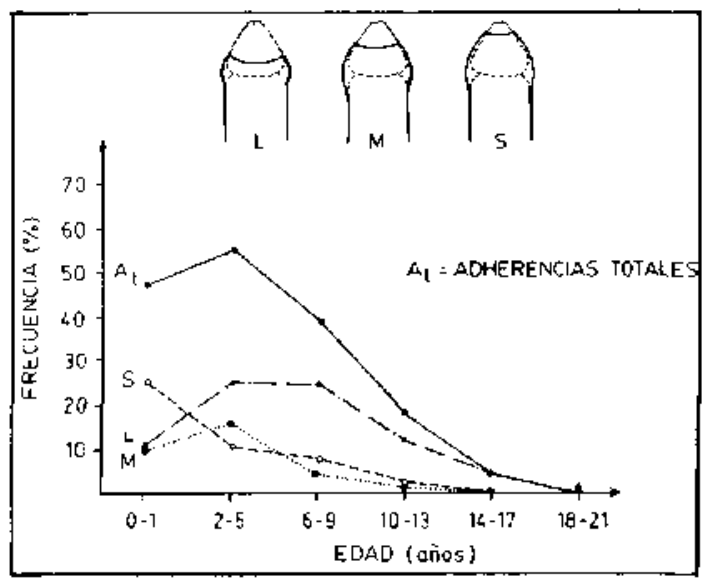

Figura 3: Evolución de las adherencias.

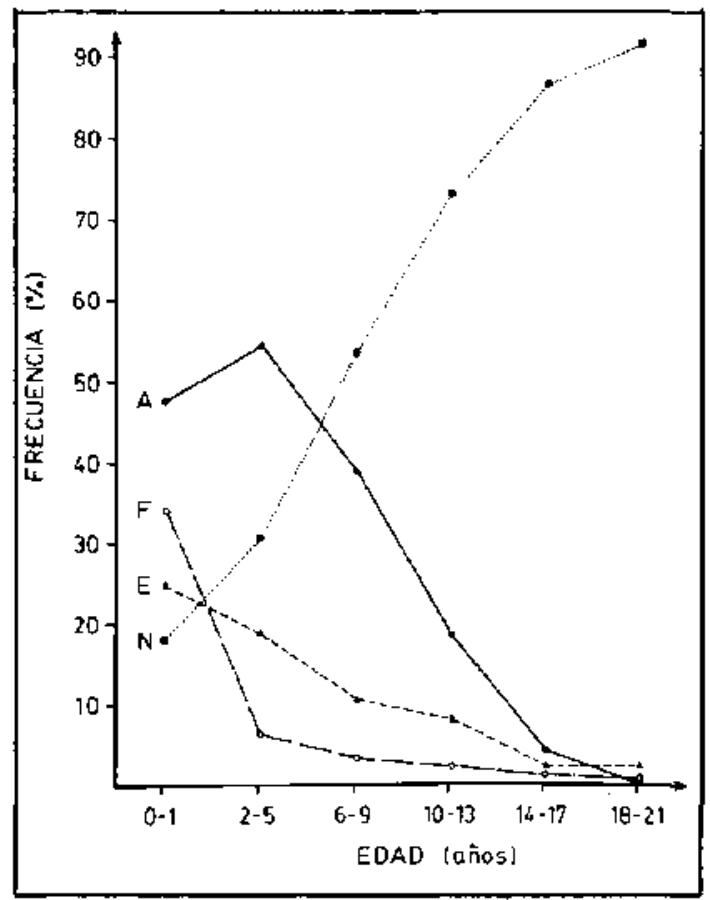

Figura 4: Evolución de la morfología prepucial. $A$ : adherencia; F: fimosis; $E$ : estrechez; $N$ : normal.

La adherencia es la motfología infantil más común, especialmente en los menores de 5 años. La fimosis predomina sobre la estrechez en el lactante, mientras que la relación se invierte en el preescolar y escolar.

\section{DISCUSION}

Los resultados obtenidos permiten caracterizar el desarrollo prepucial desde el recién nacido hasta el adolescente. Se aprecia una muy alta incidencia de fimosis y adherencia en el lactante, adherencia $y$ estrechez en el preescolar y adhe- 
rencia en el escolar. En las edades posteriores se observa una desaparición natural progresiva $y$ completa de estas características, presentando, $95 \%$. de los adolescentes de 18 a 21 años, la forma tipo adulto ( $\sin$ estrechez, ni adherencias).

La alta incidencia de adherencias encontradas, sugiere que éstas son parte de la normalidad en el desarrollo prepucial. El que éstas sean menos frecuentes en el periodo de lactante que en el preescolar, probablemente se deba a subregistro de adherencias encubiertas por fimosis. Extrapolando la curva de adherencias hacia la izquierda sin considerar su primer punto, se obtendrá que casi $100 \%$ de los recién nacidos presentan adberencias entre prepucio y glande, lo que está de acuerdo con los hallazgos histológicos de Gairdner ${ }^{2}$.

Como la fimosis y la esirechez desaparecen espontáneamente en la mayoría de los niños de 6 años, el fenómeno podría ser parte del crecimiento y desarrolio prepucial normal, por lo que parecería poco recomendable intervenir en este proceso antes de esa edad, a menos que se presente alguna afección concomitante como balanopostitis. Se puede plantear, finalmente, que existen dos fenómenos fisiológicos distintos: uno es la continuidad en los primeros años de vida de los epitelios de glande y prepucio. Se postula que éstos irían separándose por crecimiento y desarrollo y por producción de smegma. El otro, está constituido por el creci- miento prepucial, que en un comienzo es menor y retardado en relación al crecimiento del glande.

\section{RESUMEN}

Un total de 1.968 niños de 0 a 21 afios fueron examinados para determinar las características del prepucio. Se consignaron cuatro tipos morfológicos principales: "normal" (sin estrechez ni adherencia); fimosis (prepucio no retráctil); prepucio estrecho (retráctil, forma cuello en surco balano prepucial) y prepucio adherido (adherencia balano prepucial). Menos de $20 \%$ de los nirios de 0 a 23 meses presentan la forma normal, predominando las adherencias $y$ las fimosis. Durante la niñez y adolescencia se aprecia una evolución natural hacia la forma normal, observable en $50 \%$ de los niños de 7 años o $95 \%$ a los 18 años. Estas diferentes morfologías forman parte de el desarrollo prepucial normał, siendo recomendable una actitud conservadora en ausencia de complicaciones.

\section{REFERENCIAS}

1. Soriano, H.E., ORyan, M., Fanta, E. Torres, J., Muñoz, A., Soriano. H.A.: Decidir el destino del prepucio. Rey Chij Pediatr 54: 439, 1983.

2. Gaidner, D.: The fate of the foreskin. A study of circumcisión. Br Med J 2: 1433, 1949.

3. Oster, J: Further fate of the foreskin. Arch Dis Child 43; 200, 1968. 